\title{
Facoemulsificação, vitrectomia via pars plana e implante de lente intra-ocular em olhos com retinopatia diabética proliferativa
}

\author{
Phacoemulsification, pars plana vitrectomy and intraocular lens implant in eyes with \\ proliferativediabetic retinopathy
}

\author{
Armando Belfort Mattos ${ }^{1}$ \\ Pedro Paulo de Oliveira Bonomo² \\ Lincoln Lemes Freitas ${ }^{3}$ \\ Michel Eid Farah" \\ Harry Flynn Jr5 \\ Maurício Bastos Pereira ${ }^{3}$
}

Departamento de Oftalmologia da Universidade Federal de São Paulo - UNIFESP - Brasil e no "Bascom Palmer Eye Institute", hospital filiado à Universidade de Miami - Flórida - Estados Unidos da América.

Mestre em Oftalmologia pela Universidade Federal de São Paulo - UNIFESP.

${ }_{2}^{2}$ Professor Doutor em Medicina pela Universidade Federal de São Paulo - UNIFESP.

${ }^{3}$ Doutor em Medicina pela Universidade Federal de São Paulo - UNIFESP.

${ }^{4}$ Professor Livre Docente pela Universidade Federal de São Paulo - UNIFESP.

Professor do Bascom Palmer Eye Institute - Universidade de Miami - Flórida, Estados Unidos da América.

Endereço para correspondência: Rua Oscar Freire, 530 - 4ำ andar - São Paulo (SP) CEP 01426-000

E-mail: abelfortmattos@uol.com.br

Recebido para publicação em 17.07.2003

Versão revisada recebida em 21.01.2004

Aprovação em 06.02.2004

Nota Editorial: Pela análise deste trabalho e por sua anuência na divulgação desta nota, agradecemos aos Drs. Sérgio Lustosa da Cunha e Leiria de Andrade Neto.

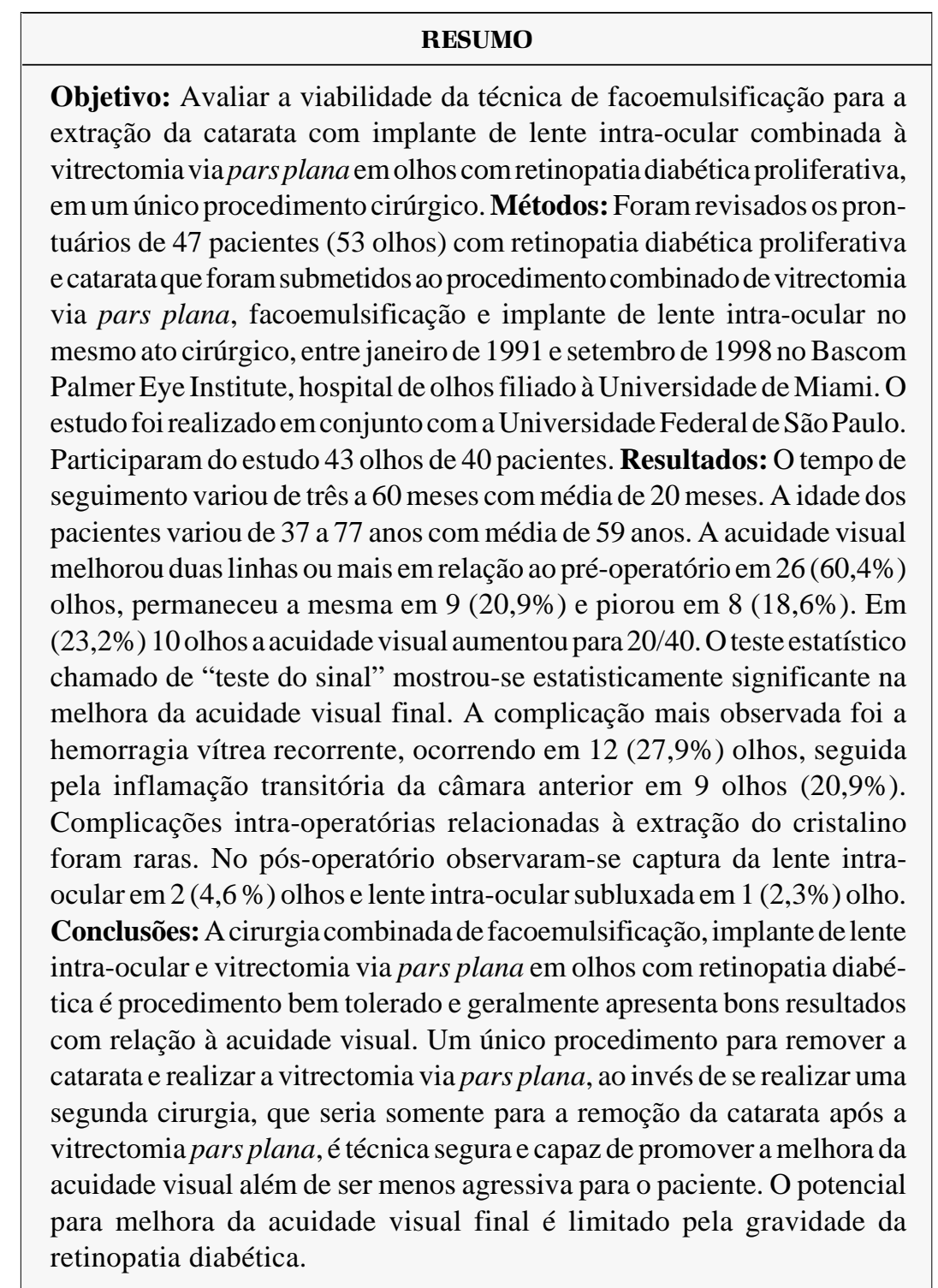

Descritores: Retinopatia diabética/cirurgia; Facoemulsificação/métodos; Extração de catarata; Lentes intra-oculares; Vitrectomia 


\section{INTRODUÇÃO}

Dominadas as técnicas cirúrgicas de vitrectomia via pars plana e de extração da catarata, começaram a surgir os primeiros trabalhos publicados envolvendo um único procedimento que combinassem as duas técnicas. Inicialmente para a extração da catarata eram utilizadas as técnicas de lensectomia via pars plana e de extração extracapsular do cristalino, por serem as mais conhecidas e de uso mais freqüente dos cirurgiões de retina $^{(1-3)}$. Posteriormente a facoemulsificação passou a ser mais utilizada a partir dos anos $90^{(4)}$.

Poucas séries de cirurgia combinada foram realizadas exclusivamente em olhos com retinopatia diabética proliferativa e geralmente relatavam um número pequeno de casos. Em 1989, utilizando a técnica de lensectomia via pars plana, tem início os primeiros trabalhos de procedimento combinado realizados exclusivamente em 21 olhos com retinopatia diabética proliferativa $^{(1)}$. Em condições semelhantes, outros autores relataram os seus resultados em séries com 15 olhos, 18 olhos e seis olhos, que apresentavam retinopatia diabética proliferativa ${ }^{(3,5-6)}$.

Em 1990 e 1992, temos as primeiras séries publicadas em que se utiliza a técnica da facoemulsificação em olhos com retinopatia diabética proliferativa ${ }^{(4,7)}$. Em 1994 e 1998, surgem as publicações com séries maiores de olhos apresentando retinopatia diabética proliferativa e que foram operados por facoemulsificação com implante de lente intra-ocular e vitrectomia via pars plana $^{(8-9)}$.

Os cirurgiões passaram a questionar o papel do cristalino nas cirurgias que envolvem a vitrectomia. Para os pacientes que irão se submeter a vitrectomia via pars plana, determinar a melhor época em que se deve retirar o cristalino e qual a melhor maneira para fazê-lo, tornou-se uma questão controversa, pois o cristalino tem relação fundamental com o prognóstico e a evolução da doença. As complicações da afacia, levando ao glaucoma neovascular, e a importância da pseudofacia com a manutenção da cápsula posterior para a prevenção do glaucoma neovascular são bem entendidas e documentadas. A progressão da retinopatia diabética após a facectomia, além da rápida progressão da catarata após a cirurgia de vitrectomia pars plana também são ressaltadas na literatura. Portanto, não seria mais fácil e menos traumático para o paciente, realizar uma única intervenção cirúrgica onde se remove a catarata e se realiza a vitrectomia, ao invés de submetê-lo a uma segunda cirurgia para a remoção da catarata após a vitrectomia? Sua recuperação visual não seria mais rápida? As visitas ao médico seriam menos freqüentes? Duas cirurgias ao invés de uma teriam melhores ou piores resultados?

A pequena literatura publicada sobre a cirurgia combinada de facoemulsificação e vitrectomia via pars plana em olhos com retinopatia diabética e os diversos questionamentos ora apresentados estimularam a realização deste trabalho que tem por objetivo avaliar os possíveis benefícios da cirurgia combinada e as suas implicações no seguimento e evolução da retinopatia diabética proliferativa, bem como estabelecer sua viabilidade e importância na cirurgia ocular moderna.

\section{MÉTODOS}

Este estudo foi retrospectivo e realizado com base na revisão dos prontuários de 47 pacientes, num total de 53 olhos com retinopatia diabética proliferativa e catarata que foram submetidos ao procedimento combinado de vitrectomia via pars plana, facoemulsificação e implante de lente intra-ocular no mesmo ato cirúrgico, entre janeiro de 1991 e setembro de 1998 no "Bascom Palmer Eye Institute", filiado à Universidade de Miami. Foram objeto de estudo 43 olhos de 40 pacientes.

Dos 53 olhos previamente selecionados, três foram excluídos devido ao pequeno tempo de seguimento e sete porque as cirurgias foram realizadas por cirurgiões não participantes do estudo e por apresentarem prontuário médico com informações incompletas.

Os critérios clínicos para que os pacientes selecionados pudessem se submeter ao procedimento combinado incluíam: pacientes com catarata e retinopatia diabética proliferativa, em que as condições anatômicas da retina fossem tecnicamente viáveis para que se pudesse realizar uma cirurgia e ter um prognóstico de melhora da acuidade visual; catarata de grau moderado a avançado, que prejudicasse a observação da retina durante a vitrectomia e dificultasse a melhora da acuidade visual no pós-operatório imediato; possibilidade de oferecer ao paciente a chance de se submeter somente a um procedimento cirúrgico, evitando uma segunda cirurgia para o implante de lente intra-ocular.

Todos os pacientes tiveram avaliações pré-operatória e pósoperatória completas. Isto incluiu medição da acuidade visual na tabela de Snellen, com a melhor correção óptica, anamnese com história de cirurgias prévias, inclusive fotocoagulação da retina, verificação da pressão intra-ocular, biomicroscopia dos segmentos anterior e posterior, classificação da catarata quanto ao grau de opacificação, em intervalo variando de: nenhuma opacidade $0+$, leve $1+$, moderada $2-3+$ e avançada $4+$.

Foi realizada a oftalmoscopia indireta para a avaliação do estado clínico da mácula e alterações vitreorretinianas. As ultra-sonografias A e B eram obtidas quando havia dificuldade de se observar toda a retina. A biometria para cálculo da lente intra-ocular foi realizada em todos os pacientes. As visitas pósoperatórias deram-se de maneira não programada previamente, com intervalos variáveis, mas sempre de acordo com a necessidade individual de cada paciente. O tempo de seguimento variou de três a 60 meses com média de 20 meses. Dos 40 pacientes no estudo, 23 eram do sexo feminino e 17 do sexo masculino. Suas idades variavam de 37 a 77 anos com média de 59 anos.

Considerou-se como melhora da acuidade visual o ganho de pelo menos duas linhas na tabela de Snellen. Também foram considerados como tendo melhora da acuidade visual os olhos que evoluíram de percepção de luz e movimento de mão para conta dedos e 6/200, ou melhor, e os olhos que evoluíram de conta dedos e de $1 / 200$ até 5/200 para 20/400, ou melhor. Não foi utilizada nenhuma tabela logarítmica para avaliar a melhora da acuidade visual. A pressão intra-ocular pósoperatória foi considerada elevada quando acima de $24 \mathrm{mmHg}$, transitória se fosse restrita ao primeiro mês. A pressão intra- 
ocular pós-operatória que persistia elevada em pacientes que ainda precisavam de qualquer tipo de medicação antiglaucomatosa até o fim do segmento no estudo foi considerada indicativa de glaucoma adquirido. A inflamação da câmara anterior foi considerada complicação quando graduada como $3+$, em intervalo de zero a 4+.

A cirurgia proposta neste artigo foi realizada por cinco cirurgiões diferentes, com vários anos de experiência em cirurgia vítreo-retiniana.

Quanto ao histórico cirúrgico dos 43 olhos participantes do estudo, $6(13,9 \%)$ foram previamente submetidos à vitrectomia pelo menos uma vez e $2(4,6 \%)$ possuíam implante escleral de silicone por 360 graus. Um olho $(2,3 \%)$ apresentava óleo de silicone como substituto do vítreo. Um total de $32(74,4 \%)$ olhos apresentava marcas de laser na retina previamente à cirurgia. No pré-operatório, 2 (4,6\%) olhos foram considerados glaucomatosos e faziam uso de medicação ocular antihipertensiva. Neovasos de íris foram diagnosticados em 5 $(11,6 \%)$ olhos e glaucoma neovascular em $2(4,6 \%)$ olhos. Na tabela 2 encontra-se uma síntese dos dados pré-operatórios.

Foram encontradas alterações maculares grave, detectadas em $19(44,1 \%)$ olhos, ou seja, edema de mácula em 2 (4,6 $\%$ ), mácula isquêmica em 2 (4,6\%), buraco macular em $2(4,6 \%)$ e mácula descolada em $13(30,2 \%)$ olhos. No exame oftalmológico pré-operatório foram observadas alterações vítreo-retinianas associadas à retinopatia proliferativa. A proliferação fibrovascular ocorreu em 7 (16,2\%) olhos, hemorragias subretinianas em 2 (4,6\%), sinéquias posteriores em 1 (2,3\%) olho e doenças vasculares da retina, como oclusão de ramo de veia central da retina em $3(6,9 \%)$ olhos e neurite óptica isquêmica aguda em $1(2,3 \%)$ olho. A catarata foi considerada de opacidade moderada em $39(90,6 \%)$ olhos e avançada em $4(9,3 \%)$. As cataratas de opacidade moderada permitiram a observação da retina com certa dificuldade pela oftalmoscopia indireta.

As principais indicações para vitrectomia foram: hemorragia vítrea persistente em $12(27,9 \%)$ olhos, descolamento tracional e/ou regmatogênico de retina em $10(23,2 \%)$, hemorragia vítrea persistente combinada com descolamento tracional e/ou regmatogênico de retina em 21 (48,8\%) olhos, sendo esta a indicação mais freqüente.

$\mathrm{Na}$ avaliação estatística deste trabalho foi utilizado o teste do sinal na avaliação da melhora da acuidade visual final ${ }^{(10)}$. O nível de rejeição para a hipótese de nulidade foi fixado em um valor igual ou menor do que 0,05 (5\%); quando a estatística calculada apresentou significância usou-se um asterisco (*) para caracterizá-la.

\section{RESULTADOS}

A acuidade visual no pré-operatório, entre os 43 olhos estudados foi de percepção de luz em cinco olhos, movimentos de mão em 11 olhos e conta dedos a diferentes distâncias inferiores a 5 metros em nove olhos. Um total de 31 (72\%) olhos apresentou acuidade visual menor ou igual a 20/200.
A acuidade visual melhorou duas linhas ou mais em relação ao pré-operatório em $26(60,4 \%)$ olhos, permaneceu a mesma em $9(20,9 \%)$ e piorou em $8(18,6 \%)$. Em $12(27,9 \%)$ olhos no préoperatório e em 21 (48,8\%) olhos no pós-operatório, a acuidade visual foi de 20/200, ou melhor. Em $10(23,2 \%)$ olhos a acuidade visual melhorou, chegando a 20/40, ou melhor.

No pós-operatório, entre os resultados ruins para acuidade visual final, $2(4,6 \%)$ olhos terminaram com percepção de luz, $4(9,3 \%)$ com movimentos de mão, $4(9,3 \%)$ com conta dedos e $5(11,6 \%)$ olhos sem percepção luminosa. Um total de $19(44,1 \%)$ olhos apresentava acuidade visual menor ou igual a 20/400. O gráfico mostra a evolução da acuidade visual.

$\mathrm{Na}$ análise dos dados estatísticos, o teste do sinal mostrou-se estatisticamente significante $(* p<0,05)$ na melhora da acuidade visual final. Do total de 43 olhos, 26 foram considerados como sinal positivo (+) e oito como sinal negativo (-). Os nove olhos em que a acuidade visual (AV) permaneceu a mesma foram excluídos do teste.

Diversos fatores de risco foram selecionados neste estudo, como os apontados por Thompson em 1986, sobre os vários indicadores prognósticos para o êxito e a falha da vitrectomia na retinopatia diabética proliferativa ${ }^{(10)}$. Acreditase que o número elevado de fatores de risco presentes tenham limitado a melhora da acuidade visual final.

Doenças afetando a mácula como descolamento, edema, isquemia e buraco, além de rubeosis iridis, baixa acuidade visual pré-operatória, descolamento de retina tracional associado à hemorragia vítrea, hipertensão arterial sistêmica, vitrectomias repetidas e o tempo de duração da cirurgia foram avaliados e considerados como prováveis fatores de pior prognóstico na melhora da acuidade visual pós-operatória. Considerando neste estudo a acuidade visual de 20/200 ou pior na tabela de Snellen, como sendo um resultado ruim para a acuidade visual final pós-operatória, os fatores prognósticos e o número de olhos em que isso ocorreu foram resumidos na tabela 1.

O tempo de duração de cada cirurgia variou de 65 a 260 minutos, com média de 151 minutos. Não ocorreram complicações relacionadas ao tempo das cirurgias, inclusive naquelas

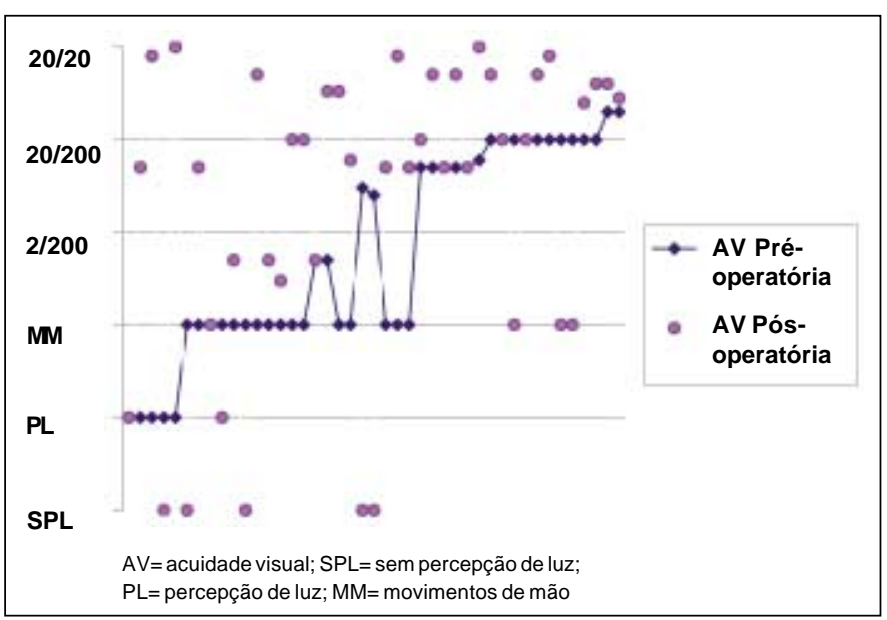

Gráfico - Comportamento da acuidade visual no pré e pós-operatório 
Tabela 1. Fatores prognósticos pré e intra-operatórios para acuidade visual final menor ou igual a 20/200

\begin{tabular}{lccc} 
Fatores prognósticos & No de olhos & AV final $\leq \mathbf{2 0 / 2 0 0}$ & Porcentagem \\
Maculopatias & 19 & 12 & $63,1 \%$ \\
DR tracional associado à HV & 21 & 13 & $61,9 \%$ \\
Vitrectomias repetidas & 11 & 2 & $72,7 \%$ \\
Rubeosis iridis & 3 & 21 & $66,6 \%$ \\
Baixa AV pré-operatória & 30 & 17 & $70,0 \%$ \\
Doenças sistêmicas associadas & 29 & 4 & $58,6 \%$ \\
Rupturas de retina per-operatórias & 8 & 8 & $50,0 \%$ \\
Tempo cirúrgico >151 minutos & 17 & & $47,0 \%$ \\
DR descolamento de retina; $\mathrm{HV}=$ hemorragia vítrea; $\mathrm{AV}=$ acuidade visual & & & \\
\hline
\end{tabular}

de maior duração. Não foram relatadas complicações associadas ao uso da anestesia local peribulbar com sedação, que pudessem ter prejudicado o ato cirúrgico.

Durante as cirurgias, foram utilizados o gás $\mathrm{C}_{3} \mathrm{~F}_{8}$ a $14 \%$ em 12 (27,9\%) olhos, óleo de silicone em 3 (6,9\%) olhos, sendo que dois olhos foram reoperações. A remoção de membranas préretinianas foi necessária em $36(83,7 \%)$ olhos. Endolaser suplementar foi aplicado em $40(93,0 \%)$ olhos. A associação de introflexão escleral à vitrectomia via pars plana ocorreu em dois olhos no pré-operatório, em três olhos no intra-operatório e em um olho que foi reoperado, ou seja, num total de $6(13,9 \%)$ olhos.

No intra-operatório observou-se hemorragia vítrea persistente em $2(4,6 \%)$ olhos e rupturas provocadas involuntariamente pelo cirurgião ou observadas somente por ocasião da vitrectomia em $8(18,6 \%)$ olhos. As rupturas foram tratadas por endofotocoagulação com laser e não foram consideradas como responsáveis pelo descolamento recorrente da retina no pós-operatório, conforme mostra a tabela 3.

A hemorragia vítrea recorrente (persistente e/ou transitória) foi a complicação mais frequiente, ocorrendo em $12(27,9 \%)$ olhos, seguida pela inflamação transitória da câmara anterior, segunda complicação mais encontrada em 9 (20,9\%) olhos. O glaucoma previamente existente em dois olhos manteve-se presente e desenvolveu-se em outros cinco olhos, num total de $7(16,2 \%)$ olhos e nenhum olho precisou ser submetido à trabeculectomia. A pressão intra-ocular foi controlada com medicamentos anti-hipertensivos, o edema macular e a isquemia da mácula foram observados em igual universo de 7 $(16,2 \%)$ olhos. A rubeosis iridis inicial regrediu em dois dos três olhos, desenvolveu-se em dois olhos, conjuntamente com o glaucoma neovascular e esteve presente no pós-operatório num total de $6(13,9 \%)$ olhos. O glaucoma neovascular presente em dois olhos no pré-operatório desenvolveu-se em mais 2 olhos, perfazendo um total de $4(9,3 \%)$ olhos no pós-operatório, conforme mostra a tabela 2 .

Um total de 17 olhos teve a lente intra-ocular de câmara posterior implantada no sulco e 26 olhos no saco capsular. Complicações intra-operatórias relacionadas à extração do cristalino foram poucas. Dentre elas, em 3 (6,9\%) olhos ocorreu diálise zonular com ruptura de cápsula posterior. Durante o implante da lente intra-ocular em um olho a alça desta lente quebrou-se e teve de ser substituída.
No pós-operatório se observou captura da lente intraocular em 2 (4,6\%) olhos, lente intra-ocular subluxada em 1 $(2,3 \%)$ olho e inflamação transitória da câmara anterior na maioria dos pacientes, mas somente foi considerada clinicamente significante em 9 (20,9\%) olhos. Aplicação de "Yag Laser" para tratar a opacidade de cápsula posterior foi necessária em $8(18,6 \%)$ olhos.

Em relação ao número de cirurgias adicionais, considerando-se vitrectomias prévias e reoperações realizadas após o procedimento combinado, encontrou-se ao fim do estudo um total de $11(25,5 \%)$ olhos submetidos a múltiplas vitrectomias, sendo que 10 olhos foram operados pelo menos duas vezes e um olho por três vezes. Procedimentos cirúrgicos secundários, como transplante de córnea, iridectomia periférica e enucleação foram necessários para reparar algumas complicações, as quais são citadas durante a evolução de cada caso, presentes na tabela 4 .

Tabela 2. Achados das complicações pós-operatórias

\begin{tabular}{lcc} 
Complicações pós-operatórias & $\begin{array}{c}\text { Número } \\
\text { de olhos }\end{array}$ & $\begin{array}{c}\text { Porcentagem do } \\
\text { total de olhos } \\
\text { estudados }\end{array}$ \\
Hemorragia vítrea & 12 & $27,9 \%$ \\
Inflamação da câmara anterior & 9 & $20,9 \%$ \\
Aumento transitório da Po & 8 & $18,6 \%$ \\
Membrana epirretiniana macular & 8 & $18,6 \%$ \\
Mácula isquêmica & 7 & $16,2 \%$ \\
Edema de mácula & 7 & $16,2 \%$ \\
Glaucoma & 7 & $16,2 \%$ \\
Descolamento de retina & 5 & $11,6 \%$ \\
Glaucoma neovascular & 4 & $9,3 \%$ \\
Sinéquias posteriores & 4 & $9,3 \%$ \\
Hipotonia & 3 & $6,9 \%$ \\
Rubeosis iridis & 2 & $4,6 \%$ \\
Captura da LIO & 2 & $4,6 \%$ \\
Phthisis & 2 & $4,6 \%$ \\
Descompensação da córnea & 2 & $4,6 \%$ \\
Endoftalmite & 1 & $2,3 \%$ \\
Hifema & 1 & $2,3 \%$ \\
Hemorragia submacular & 1 & $2,3 \%$ \\
LIO subluxada & 1 & $2,3 \%$ \\
LIO= lente intra-ocular; Po= pressão intra-ocular & \\
\hline & &
\end{tabular}




\begin{tabular}{|c|c|c|c|c|c|}
\hline Olhos & $\begin{array}{l}\text { Seguimento } \\
\text { em meses }\end{array}$ & Cirurgia realizada & $\begin{array}{c}\text { Tempo da cirurgia } \\
\text { (minutos) }\end{array}$ & Implante da LIO & $\begin{array}{l}\text { Complicações } \\
\text { intra-operatórias }\end{array}$ \\
\hline 1 & 17 & VVPP/EL/MP & 105 & SACO CAPS & Sem complicações \\
\hline 2 & 39 & VVPP/EL/MP/GÁS & 90 & SACO CAPS & Sem complicações \\
\hline 3 & 3 & VVPP/EL & 130 & SACO CAPS & Rotura de cápsula \\
\hline 4 & 12 & VVPP/EL/MP/GÁS & 205 & Sulco & Rotura de retina \\
\hline 5 & 11 & VVPP/EL/MP & 105 & SACO CAPS & Sem complicações \\
\hline 6 & 8 & VVPP/EL/MP/GÁS & 120 & Sulco & Rotura de cápsula \\
\hline 7 & 8 & VVPP/EL/MP & 135 & SACO CAPS & Sem complicações \\
\hline 8 & 12 & VVPP/EL/MP & 95 & SACO CAPS & Sem complicações \\
\hline 9 & 3 & VVPP/EL/GÁS & 130 & SACO CAPS & Sem complicações \\
\hline 10 & 13 & VVPP/EL/MP/GÁS & 185 & Sulco & Sem complicações \\
\hline 11 & 12 & VVPP/EL/MP & 130 & SACO CAPS & Rotura de retina \\
\hline 12 & 26 & VVPP/EL & 145 & Sulco & Sem complicações \\
\hline 13 & 10 & VVPP/EL/MP & 260 & SACO CAPS & Sem complicações \\
\hline 14 & 7 & VVPP/MP & 125 & Sulco & Sem complicações \\
\hline 15 & 4 & VVPP//EL/MP & 125 & Sulco & Sem complicações \\
\hline 16 & 15 & VVPP/EL/MP & 100 & SACO CAPS & Sem complicações \\
\hline 17 & 37 & VVPP/MP & 150 & Sulco & Sem complicações \\
\hline 18 & 20 & VVPP/EL/MP/GÁS & 145 & Sulco & Rotura de retina \\
\hline 19 & 47 & VVPP/EL/MP/GÁS & 165 & SACO CAPS & Sem complicações \\
\hline 20 & 48 & VVPP/EL & 180 & SACO CAPS & Sem complicações \\
\hline 21 & 46 & VVPP/EL & 200 & Sulco & Sem complicações \\
\hline 22 & 31 & VVPP/EL/MP & 210 & SACO CAPS & Sem complicações \\
\hline 23 & 26 & VVPP/EL/MP & 205 & SACO CAPS & Hemorragia vítrea \\
\hline 24 & 23 & VVPP/MP/OS & 150 & SACO CAPS & Sem complicações \\
\hline 25 & 32 & VVPP/EL/MP/GÁS & 180 & SACO CAPS & Rotura de retina \\
\hline 26 & 23 & VVPP/EL/MP & 165 & Sulco & Háptico da LIO roto \\
\hline 27 & 4 & VVPP/EL/MP & 195 & SACO CAPS & Sem complicações \\
\hline 28 & 29 & VVPP/EL/MP & 65 & SACO CAPS & Rotura de retina \\
\hline 29 & 46 & VVPP/EL/MP & 220 & Sulco & Rotura de retina \\
\hline 30 & 11 & VVPP/EL/MP & 140 & SACO CAPS & Sem complicações \\
\hline 31 & 12 & VVPP/EL/MP & 190 & Sulco & Hemorragia vítrea \\
\hline 32 & 59 & VVPP/EL/MP/GÁS & 225 & SACO CAPS & Rotura de retina \\
\hline 33 & 5 & VVPP/EL/MP & 115 & Sulco & Sem complicações \\
\hline 34 & 18 & VVPP/EL/MP/GÁS & 120 & SACO CAPS & Sem complicações \\
\hline 35 & 13 & VVPP/EL & 80 & SACO CAPS & Sem complicações \\
\hline 36 & 8 & VVPP/EL/MP & 102 & Sulco & Rotura de cápsula \\
\hline 37 & 6 & VVPP/PNEU/EL/MP & 175 & Sulco & Sem complicações \\
\hline 38 & 8 & VVPP/PNEU/EL/MP & 140 & SACO CAPS & Sem complicações \\
\hline 39 & 60 & VVPP/EL/MP & 150 & Sulco & Sem complicações \\
\hline 40 & 11 & VVPP/PNEU/EL/MP/GÁS & 143 & Sulco & Sem complicações \\
\hline 41 & 13 & VVPP/EL/MP & 190 & SACO CAPS & Rotura de retina \\
\hline 42 & 8 & VVPP/EL/MP/GÁS & 215 & SACO CAPS & Sem complicações \\
\hline 43 & 8 & VVPP/EL & 100 & SACO CAPS & Sem complicações \\
\hline
\end{tabular}

\section{DISCUSSÃO}

Diversos estudos clínicos prospectivos e novas indicações para vitrectomia vêm definindo o tratamento da retinopatia diabética nos últimos anos. Uma situação freqüente para os cirurgiões de retina são os olhos que apresentam retinopatia diabética proliferativa e catarata. A questão de extrair a catara- ta em conjunto com a vitrectomia via pars plana (VVPP) nestes olhos ainda é pouco estudada e surgiu numa tentativa de acelerar a recuperação visual do paciente, evitando a necessidade de uma segunda cirurgia.

A existência da catarata moderada em olhos com RDP que serão submetidos à vitrectomia gera duas situações: deve-se preservar o cristalino ou removê-lo implantando a lente intra- 


\begin{tabular}{|c|c|c|c|}
\hline Olhos & AV & Evolução / Complicações & Procedimentos adicionais \\
\hline 1 & $20 / 400$ & HV/Edema macular/CA INFL/Glaucoma/MEPI & Fotocoagulação \\
\hline 2 & SPL & Captura da LIO/DR/Mácula isquêmica/Phthisis & Nenhum \\
\hline 3 & PL & Mácula isquêmica/Glaucoma/MEPI & Nenhum \\
\hline 4 & SPL & RIRIS/GLNV/LIO subluxada & Nenhum \\
\hline 5 & $20 / 400$ & Mácula isquêmica & Nenhum \\
\hline 6 & $20 / 200$ & Mácula isquêmica & Nenhum \\
\hline 7 & $20 / 400$ & RIRIS/HV/Edema macular/MEPI & Nenhum \\
\hline 8 & $20 / 40$ & DR & Nenhum \\
\hline 9 & $4 / 200$ & CA INFL/Endoftalmite/Sinéquia & VVPP/Antibiótico intravitreo \\
\hline 10 & $20 / 40$ & MEPI & YAG laser \\
\hline 11 & $1 / 200$ & RIRIS/GLNV/Sinéquia & Nenhum \\
\hline 12 & $20 / 300$ & RIRIS/GLNV & Nenhum \\
\hline 13 & $20 / 200$ & HV & VVPP/Gás \\
\hline 14 & PL & CA INFL/Hipotonia/Sinéquia/Edema de córnea & VVPP/Implante de OS \\
\hline 15 & $C D$ & CA INFL & Nenhum \\
\hline 16 & $3 / 200$ & Edema macular/CA INFL/Hemorragia submacular & Fotocoagulação \\
\hline 17 & $20 / 25$ & HV/DR/Edema macular/Glaucoma & Fotocoagulação/YAG laser \\
\hline 18 & $20 / 100$ & Mácula isquêmica/MEPI & Nenhum \\
\hline 19 & $20 / 60$ & MEPI/Sinéquia & Iridectomia/YAG laser \\
\hline 20 & $20 / 25$ & 0 & Nenhum \\
\hline 21 & $20 / 60$ & HV/Edema macular/PIOTRANS/Glaucoma & YAG laser \\
\hline 22 & SPL & RIRIS/HV/DR/PIOTRANS/Hifema/Phthisis & VVPP/PNEU/Gás/EL/Enucleação \\
\hline 23 & $20 / 40$ & PIOTRANS/MEPI & Nenhum \\
\hline 24 & SPL & Captura da LIO/HV/DR/PIOTRANS/Hipotonia & VVPP/Retirada de OS \\
\hline 25 & $10 / 200$ & 0 & YAG laser \\
\hline 26 & $20 / 40$ & HV/Edema macular/CA INFL/PIOTRANS & Fotocoagulação \\
\hline 27 & $C D$ & Edema macular & Nenhum \\
\hline 28 & $20 / 50$ & 0 & Nenhum \\
\hline 29 & $20 / 40$ & HV/Glaucoma & Fotocoagulação/YAG laser \\
\hline 30 & $20 / 300$ & 0 & Nenhum \\
\hline 31 & $20 / 25$ & 0 & Fotocoagulação \\
\hline 32 & SPL & HV/Hipotonia & Nenhum \\
\hline 33 & $2 / 200$ & RIRIS/CA INFL/GLNV & VVPP/OS \\
\hline 34 & $3 / 200$ & Sinéquia & YAG laser \\
\hline 35 & $20 / 80$ & 0 & Nenhum \\
\hline 36 & $20 / 20$ & 0 & Nenhum \\
\hline 37 & $6 / 200$ & 0 & Nenhum \\
\hline 38 & $20 / 300$ & Edema corneano & Transplante de córnea \\
\hline 39 & $20 / 20$ & PIOTRANS/CA INFL & YAG laser \\
\hline 40 & $20 / 70$ & PIOTRANS/CA INFL & Nenhum \\
\hline 41 & $20 / 50$ & HV/Glaucoma & Nenhum \\
\hline 42 & $20 / 200$ & Mácula isquêmica/Glaucoma/MEPI & Fotocoagulação \\
\hline 43 & $20 / 200$ & HV/PIOTRANS/Mácula isquêmica/MEPI & Nenhum \\
\hline \multicolumn{4}{|c|}{$\begin{array}{l}\mathrm{AV}=\text { acuidade visual; } \mathrm{PL}=\text { percepção de luz; } \mathrm{SPL}=\text { sem percepção de luz; } \mathrm{CD}=\text { conta dedos; } \mathrm{VVPP}=\text { vitrectomia via pars plana; } \mathrm{PNEU}=\text { pneu de silicone; } \mathrm{OS}=\text { óleo } \\
\text { de silicone; } \mathrm{HV}=\text { hemorragia vítrea; } \mathrm{DR}=\text { descolamento da retina; } \mathrm{RIRIS}=\text { rubeosis iridis; } \mathrm{GLNV}=\text { glaucoma neovascular; } \mathrm{LIO}=\text { lente intra-ocular; } \mathrm{PIO} \mathrm{TRANS}=\text { aumento } \\
\text { transitório da pressão intra-ocular; } \mathrm{CA} I \mathrm{NFL}=\text { reação inflamatória transitória da câmara anterior; } \mathrm{MEPI}=\text { membrana epiretiniana; } E \mathrm{EL}=\text { endolaser }\end{array}$} \\
\hline
\end{tabular}

ocular? Para avaliar qual dessas situações é a mais adequada, algumas considerações devem ser analisadas:

Situação 1: vitrectomia via pars plana combinada com extração do cristalino. A afacia no diabético torna-se interessante exclusivamente quando se necessita realizar vitrectomia posterior em olhos com retinopatia proliferativa anterior, ocasião em que a remoção do tecido proliferativo na base vítrea, zônula ou cápsula posterior é primordial para o êxito da cirurgia ${ }^{(11)}$. Em
1996, surge artigo relatando que a afacia permite excelente observação da retina durante a VVPP, sem interferência da LIO ou da cápsula posterior ${ }^{(12)}$. Seen e colaboradores em 1995 correlacionaram o índice reduzido de acidentes durante as cirurgias devido a maior visibilidade do fundo de olho após a retirada do cristalino $^{(13)}$. A pseudofacia também permite a boa observação da retina no per e pós-operatórios. Diversos autores que realizaram a cirurgia combinada de vitrectomia via pars plana, facecto- 
mia e implante de LIO, relataram não ter dificuldades referentes ao implante da LIO e sempre mantiveram boa visibilidade da retina no per-operatório e no pós-operatório para exames e aplicações de laser ${ }^{(3,7,14)}$. Trabalhos mais recentes mostraram que ocorre rápida progressão da catarata após a vitrectomia via pars plana em período próximo de até dois anos, principalmente quando foi utilizado óleo de silicone ou gás. Cerca de $51 \%$ a $88 \%$ dos olhos desenvolvem catarata no pós-operatório. Isto faz com que o procedimento combinado exija estudo acurado como uma técnica alternativa para aqueles pacientes com retinopatia diabética proliferativa, fácicos, com esclerose do cristalino e que precisam de vitrectomia via pars plana ${ }^{(13,15-16)}$.

Situação 2: vitrectomia via pars plana sem extração da catarata. A manutenção do cristalino em olhos diabéticos com catarata moderada durante a vitrectomia via pars plana também apresenta algumas vantagens. A afacia é uma situação indesejada para pacientes diabéticos, devido ao seu alto índice de complicações, especialmente a rubeosis iridis e o glaucoma neovascular ${ }^{(17-19)}$. Em 1996, foi publicado artigo de revisão, no qual afirmava-se que a afacia, sem implante de LIO após a vitrectomia posterior, somente encontraria indicações quando as doenças do segmento posterior fossem muito graves e até mesmo a presença de restos de cápsula de cristalino seria considerada prejudicial ao olho ${ }^{(20)}$. A manutenção do cristalino para preservar a barreira física entre os segmentos anterior e posterior é preconizada por ser, entre as possíveis situações, a mais próxima da fisiológica e por evitar as complicações da afacia e da pseudofacia ${ }^{(19,21-22)}$. Apesar de haver a necessidade de um novo procedimento em um espaço curto de tempo e de ser tecnicamente mais difícil, o número de complicações per e pós-operatórias quando se realiza a facoemulsificação após a vitrectomia via pars plana é semelhante quando comparadas às complicações encontradas na cirurgia de facoemulsificação combinada com a vitrectomia via pars plana ${ }^{(23-24)}$.

O debate sobre a importância do cristalino é necessário para que possamos avaliar quando a remoção da catarata e o implante de lente intra-ocular combinados com a vitrectomia via pars plana possam fazer parte do planejamento cirúrgico dos olhos com RDP. Deve-se levar em consideração a presença ou ausência de fatores de risco e conhecer as vantagens e desvantagens do procedimento. Abaixo são comentados os resultados do nosso estudo e da presença de fatores de risco.

$\mathrm{O}$ tempo prolongado das cirurgias de procedimento combinado, o qual foi considerado como maior do que 151 minutos, seria uma desvantagem nesta situação pelo desgaste que é gerado no cirurgião e no paciente. No trabalho em questão apesar de estar relacionado como fator de risco, não há dados na literatura que atestem que o tempo de cirurgia tem influência no resultado. Senn e colaboradores em 1995, não encontraram diferenças na melhora da acuidade visual, quando compararam o tempo cirúrgico da cirurgia combinada e o da extração de catarata como procedimento secundário depois da vitrectomia via pars plana ${ }^{(13)}$.

As rupturas de retina provocadas acidentalmente pelos cirurgiões no per-operatório não foram muitas. Acredita-se que a extração do cristalino, o implante da LIO e o aperfeiçoamento das técnicas e instrumentos de vitrectomia permitiram a boa observação da retina no per e pós-operatórios, e a formação de um menor número de rupturas, apesar de freqüentes, contribuíram para um procedimento cirúrgico mais seguro e para um bom seguimento no pós-operatório, inclusive permitindo a fotocoagulação da retina. Neste estudo as rupturas iatrogênicas também foram relacionadas como fator de risco, mas não há dados suficientes na literatura que comprovem que a sua presença impeça o êxito da cirurgia combinada ${ }^{(25)}$.

Um fator de risco importante é o descolamento de retina tracional isolado ou combinado com o descolamento regmatogênico e a hemorragia vítrea. No atual estudo, 61,9\% dos olhos com descolamento de retina tracional e/ou regmatogênico evoluíram com acuidade visual menor do que 20/200. Outros autores em 1983 encontraram índices de 70\% e 75\% referentes à retina e à mácula estarem coladas ao fim do estudo ${ }^{(26)}$. Em 1989, também se registraram índices de $83 \%$ e $88 \%$ respectivamente para a retina e mácula coladas ao fim do estudo, usando técnicas como a excisão em bloco ${ }^{(27)}$. Mesmo com esse elevado índice anatômico de êxito, estes autores observaram melhora limitada da acuidade visual devido a alterações funcionais irreversíveis da retina.

A cirurgia de facoemulsificação em olhos diabéticos com a preservação da cápsula posterior e o implante de LIO é bem aceita na literatura e não é correlacionada a alta incidência de glaucoma neovascular, como ocorre na afacia ${ }^{(17,28)}$. Quando a extração da catarata é realizada por facoemulsificação e o olho apresenta rubeosis iridis no pré-operatório, observa-se que a presença da rubeosis iridis é estatisticamente significante como fator de risco para uma melhora da acuidade visual ${ }^{(10)}$. A rubeosis iridis não deve ser um fator que contra-indique o procedimento combinado, pois esta pode até regredir em certos casos. Em 1982, observa-se a regressão de rubeosis iridis em 55\% dos pacientes com RDP depois de se realizar a vitrectomia via pars plana com endolaser e aplicar a retina no pós-operatório ${ }^{(29)}$. Em 1998 surge artigo publicado em que se realizou a extração de catarata e o implante de lente intra-ocular em 35 olhos diabéticos com rubeosis iridis e observou-se que não houve complicações graves no per ou pósoperatório recente ${ }^{(30)}$.

Neste estudo houve a ruptura da cápsula posterior em 3 $(6,9 \%)$ olhos, a regressão da rubeosis iridis em dois dos três olhos no pré-operatório, descolamento de retina em $5(11,6 \%)$ e aplicação de fotocoagulação complementar realizada em 36 $(83,7 \%)$ olhos. A capsulotomia realizada por "Yag Laser" em oito olhos não influenciou na acuidade visual final. Somente dois olhos apresentaram acuidade visual menor que 20/400 e nenhum deles apresentou rubeosis iridis.

Assim como o autor deste artigo, diversos outros também relatam em seus artigos acreditarem que a manutenção da cápsula posterior do cristalino, o uso do endolaser e a manutenção da retina aplicada no pós-operatório, são os mecanismos responsáveis pela diminuição da incidência de rubeosis iridis e do glaucoma neovascular para a retinopatia diabética proliferativa ${ }^{(3,26,29)}$. O elevado número de reoperações até o fim 
do estudo, ou seja, 25,5\% dos olhos, foi ocasionado pela gravidade da retinopatia e pela tentativa de controlá-la. A necessidade de reoperações é um fator de risco importante para a falha das vitrectomias via pars plana em olhos com retinopatia diabética proliferativa. Em artigo publicado em 1992, os autores ressaltaram a alta morbidade dos olhos que requerem vitrectomias via pars plana de repetição ${ }^{(31)}$. Nesse estudo, $46 \%$ dos olhos reoperados por complicações da retinopatia diabética proliferativa apresentaram-se como com percepção de luz ou sem percepção de luz, e 44\% tiveram descolamento de retina ao fim do estudo. A utilização de substitutos vítreos pode ser necessária em casos de retinopatia proliferativa com descolamento de retina e proliferação vitreorretiniana grave, na tentativa de aplicar a retina. A utilização de gás $\mathrm{C}_{3} \mathrm{~F}_{8}$ em 12 (27,9\%) olhos, óleo de silicone em 3 (6,9\%) e índice de reoperações de $25,5 \%$, refletem a gravidade dos casos apresentados.

Em nosso estudo a hemorragia vítrea foi a principal complicação pós-operatória seguida pela reação inflamatória da câmara anterior. Estas complicações, bem como o aumento da pressão intra-ocular, são presenças marcantes em todas as séries estudadas e devem ser objeto de atenção para os cirurgiões de retina e vítreo. A hemorragia vítrea é, talvez, mais freqüente nos dias atuais do que era no início das cirurgias de vitrectomia $^{(32)}$. Isso dar-se-ia provavelmente pelo fato de se operar olhos com retinopatia proliferativa ativa e extensa remoção de membranas e áreas de tração. Já em outros estudos, a reação inflamatória da câmara anterior é citada como a principal complicação pós-operatória ${ }^{(5-6,8)}$.

A presença de acuidade visual baixa, menor que $5 / 200$, no pré-operatório como fator de risco para a melhora da acuidade visual pós-operatória em olhos com RDP é citada em $1986^{(10)}$. Artigo publicado em 1993 relata que a melhora da acuidade visual após a cirurgia de extração da catarata em olhos com RDP geralmente é limitada ${ }^{(28)}$. A gravidade da retinopatia diabética foi o fator mais importante de previsibilidade para um mau prognóstico.

Em 1994 foi publicado um artigo com uma série de 120 olhos com RDP, sendo que 57 eram do grupo de lensectomia, 49 do grupo de facoemulsificação e apenas 14 do grupo de extração extracapsular ${ }^{(8)}$. A acuidade visual final melhorou na maioria dos pacientes e somente 13 olhos apresentaram acuidade visual pior ou igual a conta dedos a menos de 5 metros ou superior. Outra série foi publicada em 1998, com 76 olhos operados por meio da cirurgia combinada, utilizando a facoemulsificação para a extração do cristalino em olhos com RDP. A acuidade visual melhorou duas linhas em $78 \%$ dos olhos ${ }^{(9)}$.

No presente estudo, a melhora da acuidade visual final igual ou superior a duas linhas na tabela de Snellen é a menor encontrada nas séries publicadas, apesar do bom índice encontrado de $60,4 \%$ dos olhos. Alguns autores afirmam que a melhora da acuidade visual está relacionada diretamente com o grau de comprometimento da retina, pela retinopatia diabética proliferativa, bem como com a pré-seleção dos pacientes para este tipo de procedimento ${ }^{(23)}$.
Os trabalhos publicados sobre procedimento combinado em olhos com retinopatia diabética proliferativa revelam que é possível obter melhora da acuidade visual em $76,1 \%$ a $100 \%$ dos casos. Os fatores de risco e as complicações neste estudo são semelhantes nos estudos em que foi realizada a vitrectomia via pars plana em olhos com RDP. A facoemulsificação com implante de lente intra-ocular de câmara posterior nunca foi considerada como fator de piora da acuidade visual no olho diabético, muito pelo contrário, é sempre necessária a remoção da catarata para tentar melhorar a acuidade visual. A facoemulsificação elimina os sérios problemas de afacia e suas conseqüências.

\section{CONCLUSÕES}

A nosso ver a cirurgia combinada proposta neste estudo é a mais adequada para tratar os olhos de diabéticos com catarata que necessitem de vitrectomia via pars plana como tratamento. A tendência para o cristalino desenvolver rapidamente a catarata no pós-operatório de vitrectomias e a necessidade de se remover o cristalino para executar melhor a vitrectomia via pars plana, além da melhora e da rápida reabilitação da acuidade visual, sem a necessidade de um segundo procedimento, são fatores que fazem esta cirurgia combinada muito atrativa, ajudando inclusive a solucionar possíveis problemas de ordem social que possam existir.

A gravidade da doença estudada faz com que os resultados para a melhora da acuidade visual sejam limitados, porém os estudos sobre o tratamento da retinopatia diabética vêm contribuindo para que essa situação possa ser modificada.

\section{ABSTRACT}

Purpose: To evaluate the outcomes and the best technique for a combined phacoemulsification (PHACO), pars plana vitrectomy (PPV) and posterior chamber intraocular lens insertion (PCIOL) in one single procedure for patients with proliferative diabetic retinopathy. Methods: We reviewed charts of 47 (53 eyes) patients with proliferative diabetic retinopathy who underwent combined phacoemulsification with posterior chamber intraocular lens implant and pars plana vitrectomy performed between January 1991 and September 1998 at the Bascom Palmer Eye Institute, eye hospital affiliated with the University of Miami. The study was done in conjunction with the Federal University of São Paulo. A total of 43 eyes from 40 patients were elected to participate in the study. Results: The follow-up range was three to 60 months (mean 20 months). The age ranged from 37 to 77 years with a mean of 59. Preoperative visual acuity improved two lines or more in $26(60.4 \%)$ eyes, remained the same in $9(20.9 \%)$ and got worse in 8 (18.6\%). In 10 (23.2\%) eyes visual acuity improved to $20 / 40$. The study showed to be statistically significant for the improvement of the final visual acuity. Recurrent vitreous hemorrhage was the most frequent postoperative complication found in $12(27.9 \%)$ eyes and it was followed by transient 
anterior chamber reaction in $9(20.9 \%)$ eyes. Intraoperative and postoperative complications related to phacoemulsification were rare. IOL capture was found in $2(4.6 \%)$ eyes and intraocular lens subluxation in $1(2.3 \%)$ eye. Conclusion: Combined phacoemulsification with posterior chamber intraocular lens implant and pars plana vitrectomy in proliferative diabetic retinopathy is a feasible procedure, well-tolerated and usually presents significant visual acuity improvement. One single procedure, to remove the cataract and to perform pars plana vitrectomy, instead of performing a second surgery that would be only to remove the cataract after the pars plana vitrectomy is safe, improves visual acuity and is also less aggressive for the patient. The potential for improving final visual acuity is limited by the severity of retinopathy.

Keywords: Diabetic retinopathy/surgery; Phacoemulsification/ methods; Cataract extraction; Lenses, intraocular; Vitrectomy

\section{REFERÊNCIAS}

1. Blankenship GW. Posterior chamber intraocular lens implantation during pars plana lensectomy and vitrectomy for diabetic complications. Graefes Arch Clin Exp Ophthalmol 1989;227:136-8.

2. Blankenship GW, Flynn HW Jr, Kokame GT. Posterior chamber intraocular lens insertion during pars plana lensectomy and vitrectomy for complications of proliferative diabetic retinopathy. Am J Ophthalmol 1989;108:1-5.

3. Kokame GT, Flynn HW Jr, Blankenship GW. Posterior chamber intraocular lens implantation during diabetic pars plana vitrectomy. Ophthalmology 1989;96:603-10.

4. Koenig SB, Han DP, Mieler WF, Abrams GW, Jaffe GJ, Burton TC. Combined phacoemulsification and pars plana vitrectomy. Arch Ophthalmol 1990;108:362-4

5. Pagot V, Gazagne C, Galiana A, Giraud MA, Malecaze F, Mathis A. Extraction extracapsulaire du cristallin et implantation dans le sac capsulaire au cours de la vitrectomie chez le diabétique. J Fr Ophthalmol 1991;14:523-8.

6. Menchini U, Azzolini C, Camesasca FI, Brancato R. Combined vitrectomy, cataract extraction, and posterior chamber intraocular lens implantation in diabetic patients. Ophthalmic Surg 1991;22:69-73.

7. Koenig SB, Mieler WF, Han DP, Abrams GW. Combined phacoemulsification, pars plana vitrectomy, and posterior chamber intraocular lens insertion. Arch Ophthalmol 1992;110:1101-4.

8. Ogino N, Uchida H. Combined pars plana vitrectomy, lens removal and intraocular lens implantation for complications of diabetic retinopathy. Surgical results in 120 cases. Nippon Ganka Gakkai Zasshi 1994;98:672-8.

9. Honjo M, Ogura Y. Surgical results of pars plana vitrectomy combined with phacoemulsification and intraocular lens implantation for complications of proliferative diabetic retinopathy. Ophthalmic Surg Lasers 1998;29:99-105.

10. Thompson JT. Auer CL, de Bustros S, Michels RG, Rice TA, Glaser BM. Prognostic indicators of success and failure in vitrectomy for diabetic retinopathy. Ophthalmology 1986;93:290-5.
11. Lewis H, Aaberg TM. Anterior proliferative vitreoretinopathy. Am J Ophthalmol 1988;105:277-84.

12. Hurley C, Barry P. Combined phacoemulsification, pars plana vitrectomy, and intraocular lens implantation. J Cataract Refract Surg 1996;22:462-6.

13. Senn P, Schipper I, Perren B. Combined pars plana vitrectomy, phacoemulsification, and intraocular lens implantation in the capsular bag: a comparison to vitrectomy and subsequent cataract surgery as a two-step procedure. Ophthalmic Surg Lasers 1995;26:420-8.

14. Mamalis N, Teske MP, Kreisler KR, Zimmerman PL, Crandall AS, Olson RJ. Phacoemulsification combined with pars plana vitrectomy. Ophthalmic Surg 1991;22:194-8.

15. Smiddy WE, Stark WJ, Michels RG, Maumenee AE, Terry AC, Glaser BM. Cataract extraction after vitrectomy. Ophthalmology 1987;94:483-7.

16. Pang MP, Peyman GA, Minatoya HK. Posterior chamber lens implantation following pars plana lensectomy and vitrectomy in severe proliferative diabetic retinopathy. Can J Ophthalmol 1989;24:175-8.

17. Aiello LM, Wand M, Liang G. Neovascular glaucoma and vitreous hemorrhage following cataract surgery in patients with diabetes mellitus. Ophthalmology 1983;90:814-20.

18. Blankenship GW. The lens influence on diabetic vitrectomy results. Report of a prospective randomized study. Arch. Ophthalmol 1980;98:2196-8.

19. Poliner LS, Christianson DJ, Escoffery RF, Kolker AE, Gordon ME. Neovascular glaucoma after intracapsular and extracapsular cataract extraction in diabetic patients. Am J Ophthalmol 1985;100:637-43.

20. Ryan EH Jr, Gilbert HD. Lensectomy, vitrectomy indications, and techniques in cataract surgery. Curr Opin Ophthalmol 1996;7:69-74.

21. Sebestyen JG. Intraocular lenses and diabetes mellitus. Am J Ophthalmol 1986;101:425-8.

22. Novak MA, Rice TA, Michels RG, Auer C. The crystalline lens after vitrectomy for diabetic retinopathy. Ophthalmology 1984;91:1480-4.

23. Grusha YO, Masket S, Miller KM. Phacoemulsification and lens implantation after pars plana vitrectomy. Ophthalmology 1998;105:287-94.

24. MCDermott ML, Puklin JE, Abrams GW, Eliott D. Phacoemulsification for cataract following pars plana vitrectomy. Ophthalmic Surg Lasers 1997;28: 558-64.

25. Machemer R, Blankenship G. Vitrectomy for proliferative diabetic retinopathy associated with vitreous hemorrhage. Ophthalmology 1981;88:643-6.

26. Schachat AP, Oyakawa RT, Michels RG, Rice TA. Complications of vitreous surgery for diabetic retinopathy II. Postoperative complications. Ophthalmology 1983;90:522-30

27. Williams DF, Williams GA, Hartz A, Mieler WF, Abrams GW, Aaberg TM. Results of vitrectomy for diabetic traction retinal detachments using the en bloc excision technique. Ophthalmology 1989;96:752-8.

28. Benson WE, Brown GC, Tasman W, McNamara JA, Vander JF. Extracapsular cataract extraction with placement of a posterior chamber lens in patients with diabetic retinopathy. Ophthalmology 1993;100:730-8.

29. Scuderi JJ, Blumenkranz MS, Blankenship G. Regression of diabetic rubeosis iridis following successful surgical reattachment of the retina by vitrectomy. Retina 1982;2:193-6.

30. Küchle M, Handel A, Naumann GO. Cataract extraction in eyes with diabetic iris neovascularization. Ophthalmic Surg Lasers 1998;29:28-32.

31. Brown GC, Tasman WS, Benson WE, McNamara JA, Eagle RC Jr. Reoperation following diabetic vitrectomy. Arch Ophthalmol 1992;110:506-10.

32. Oyakawa RT, Schachat AP, Michels RG, Rice TA. Complications of vitreous surgery for diabetic retinopathy. I. Intraoperative complications. Ophthalmology 1983;90:517-21.

\section{Ao enviar um artigo para publicação, leia ATENTAMENTE as instruções para autores, constante no final de cada fascículo.}

